\title{
Impact of Different Forms of Environmental Enrichment on Foraging and Activity Levels in Gorillas (Gorilla gorilla gorilla)
}

\author{
Kristie Charmoy $^{1}$, Tim Sullivan ${ }^{1}$, \& Lance J. Miller ${ }^{1 *}$ \\ ${ }^{1}$ Chicago Zoological Society- Brookfield Zoo \\ *Corresponding author (Email: lance.miller@czs.org)
}

Citation - Charmoy, K., Sullivan, T., \& Miller, L. J. (2015). Impact of different forms of environmental enrichment on foraging and activity levels in gorillas (Gorilla gorilla gorilla). Animal Behavior and Cognition, 2(3), 233-240. doi: 10.12966/abc.08.03.2015

\begin{abstract}
The maintenance of species-specific behaviors for animals in zoological institutions is of top priority, as this can help ensure high levels of animal welfare. Strict feeding schedules within institutions can often impact natural foraging behaviors of animals, as they are no longer required to seek out or manipulate food items. In the wild, western lowland gorillas would spend a majority of their time foraging. The goal of the current study was to examine the impact of different forms of environmental enrichment on activity and foraging levels in gorillas at the Brookfield Zoo. Results suggest that automatic belt feeders that can feed at randomized times, will have the largest impact on behavior of all enrichment tested. However, there were individual differences observed between animals and the level of impact on their behavior. Using enrichment to increase the amount of time that zoo-housed gorillas spend searching for, acquiring, and consuming food can increase their overall activity levels and shift their behavior towards a more naturalistic direction.
\end{abstract}

Keywords - Animal welfare, Gorilla, Environmental enrichment, Randomized feeding

In the wild, Western lowland gorillas live in lowland rainforests and are much more frugivorous than their mountain gorilla counterparts. Morphological differences in the shearing crests, size of incisors, and suspensory features of the scapula can explain for these differences in diet (Doran \& Mcneilage, 1998). Western lowland gorillas dedicate approximately $67-70 \%$ of their day foraging and roughly $21 \%$ of their time resting (Magliocca \& Gautier-Hion, 2002; Masi, Cipoletta, \& Robbins, 2008;). Western lowland gorillas incorporate roughly a hundred different species of fruit in their diet year-round (Doran \& McNeilage 1998; Magliocca \& Hion, 2002). Fruit consumption can influence the amount of time a species dedicates to foraging, traveling, and even socialization (Masi et al., 2008). Having a good understanding of a species natural-history when developing an enrichment program can help ensure that the animals' needs are met helping to ensure good animal welfare.

Like their wild counterparts, zoo-housed mammals are constantly adjusting their behavior in order to better adapt to their environment (McPhee \& Carlstead, 2010). Adjusting to structured feeding schedules, fixed social pairings, or even the ability to acclimate to constant visitor presence is important within this type of environment. Compared to their wild counterparts, which consume small quantities of food over large amounts of time, zoo gorillas are often restricted by animal care schedules and feeding protocols (Lukas, 1999). Gorillas housed at zoological institutions spend approximately 20-25\% of their time foraging and roughly 54\% of their time at rest (Less, Kuhar, Dennis, \& Lukas, 2012; Lukas, 1999;). 
Maintaining natural behaviors in animals is a top priority for zoos, as the presences of species-specific behaviors can be an indicator of good animal welfare (McPhee \& Carlstead, 2010).

Dawkins (2004) proposes that animal welfare can be determined by answering two important questions: is the animal healthy and does the animal have what it wants? By using behavior as a means for gauging welfare, it provides a non-invasive look into an animals' choices and indicators of what they want (Dawkins, 2004). Though a gorilla in a zoological institution is provided with the necessary food and nourishment to survive, there still may be a motivation to forage and explore their environment. Providing environmental enrichment to increase behavioral diversity in zoo animals is one method in improving animal welfare (McPhee \& Carlstead, 2010). Enrichment can typically be broken down into various categories, e.g., foraging, sensory, social, etc., based on goals and effects (Clark, 2011). These categories can often overlap and be used together in order to reach optimal stimulation and animal welfare.

In the wild, foraging is a process including the searching, acquiring, and consuming of food items. However, this process is often simplified in zoo settings, rarely requiring zoo animals to do more than simply consume (Hosey, 2005; McPhee \& Carlstead, 2010). Modifications to an animal's feeding method, intended to increase foraging times, can increase activity levels and significantly shift behavior towards a more naturalistic direction (Hosey, 2005). Providing a variety of enrichment not only promotes natural behaviors, but allows for a healthier environment and for individual preference (Rooney \& Sleeman, 1998). In order to achieve this, an enrichment program was developed to target species-specific behaviors, specifically foraging. The goal of the current study was to examine the impacts of different forms of environmental enrichment on the activity and foraging levels of gorillas.

\section{Method}

\section{Subjects}

The subjects included three adult female western lowland gorillas, G1 (26 years old), G2 (19), and G3 (10), and one adult silverback, G4 (34). G2 had a one year old infant that was not part of the study. The subjects inhabited an indoor exhibit between 10:00 and 17:00, approximately 6,400 $\mathrm{ft}^{2}$. The exhibit contained a central elevated island, man-made trees and boulders, ropes, and a waterfall that flowed into a river. The gorillas were fed a morning meal on exhibit at 10:00, a mid-day meal of kale on exhibit at approximately 12:15, and were shifted off exhibit at 15:00 for roughly 10 min for a mid-day training session.

\section{Enrichment Modification}

In addition to pre-existing environmental enrichment (e.g., nesting material, scattered diet, etc.), one new form of enrichment was introduced each week to the troop. Each week, starting September $15^{\text {th }}$, 2014, enrichment was introduced in varying degrees of difficulty and reward to encourage animals to continuously explore their exhibit. Each new treatment was added in addition to the previous weeks' treatment. Week (1) frozen blocks of koolaid or orange juice were placed inside an enrichment stump with various treats frozen inside, acting as a time released treat. Juice was frozen inside a $64 \mathrm{oz}$. polypropylene plastic container, with various dried treats (e.g., sunflower seeds, cheerios, and raisins). The frozen block was placed inside of the enrichment stump prior to the gorillas shifting onto exhibit. Week (2) keepers varied the times that gorillas were fed their mid-day kale as well as the time they were taken off exhibit for a short training session. Prior to week 2, the troop was provided kale at 12:15, at which time keepers would walk along the perimeter of the exhibit and throw allotted amount of kale to the gorillas. The troop was also taken off exhibit around 15:00, for a short husbandry-based training session. During week (2) keepers varied the time of kale-throw and exhibit shift to remove predictability, keepers were able to feed and/or shift animals between 11:00 and 15:00. This allowed for a large enough interval to remove predictability, but still allow for the animals to still be properly motivated to shift back 
off exhibit at 17:00. Week (3) an additional training session was added each day using training panels on exhibit. The gorilla training program operates under both the Chicago Zoological Society animal training guidelines as well as species-specific gorilla training protocols. All training is performed using positive reinforcement and utilized as a form of enrichment by providing cognitive stimulation as well as allowing animals' to aid in their own medical/husbandry management. Training sessions occur both on and off exhibit on a daily basis. Structured off-exhibit training includes one morning session and one mid-day session. Additional training sessions occurred between 11:00 and 15:00. Keepers utilized a small window panel located at the south end of the exhibit. The keeper would call for the troop. When a gorilla would approach the training panel, the keeper would ask for a behavior, and reward the gorilla with dried treats (e.g., raisins and peanuts). Week (4) two automatic belt feeders containing cardboard tubes with various treats inside were added to the exhibit. Automatic belt feeders were attached at the north and southwest end of the exhibit. The belt ran on a $12 \mathrm{hr}$ timer, depositing tubes into the exhibit randomly throughout the day. Eight tubes were loaded into each automatic belt feeder prior to the troops' shift onto exhibit. The tubes contained dried treats (e.g., cheerios, raisins, sunflower seeds, hay, smeared peanut butter, and koolaid powder). In order to decrease predictability, some tubes were also left empty. Week (5) additional tubes were added to each automatic belt feeder, increasing from eight to twelve tubes per automatic belt feeder. The addition of scents to random tubes was also utilized. Scents used included Estee Lauder "cinnabar" and "pleasures" as well as Acqua di Parma "colonia." Week (6) included the addition of plastic bark-boards, made to look like natural tree bark, with drilled holes containing treats. Six barkboards were placed throughout the exhibit prior to the gorillas' initial shift onto exhibit. The boards were approximately $1 \mathrm{ft} \times 2 \mathrm{ft}$, each uniquely designed. The boards were constructed using $1 / 2$ in starboard, and the drilled holes ranged from approximately $1 / 2-2 \frac{1}{2}$ in diameter filled with either a smear of peanut butter or jam.

\section{Observations and Analysis}

Behavior of the gorillas was recorded using instantaneous sampling (Altmann, 1974). Observations on each gorilla were conducted at one-minute intervals for a total of five minutes, between the hours of 10:00 - 16:00. Observations were recorded once an hour per animal, Monday through Friday. No two gorillas were observed at the same time. Observation times within each hour were generated at random using the random number generator in Microsoft Excel. Enrichment treatments introduced during this study were not provided on the weekends, however pre-existing enrichment was continued. In total, each gorilla was observed for 840 min throughout the six week period. Table 1 displays the ethogram used in the current study. All variables were created accounting for percentage of time visible and these included forage, active and abnormal. Percentage of time active included only species-specific active behaviors. Active behaviors included: foraging, locomotion, self-maintenance, solitary play, and social behaviors. All data were examined for normality. Differences in activity and foraging levels due to the environmental enrichment were examined using a Friedman's two-way analysis. Due to small sample size, Wilcoxon sign-rank tests to follow up for significant differences could not be utilized. An alpha value was set at $p<0.05$ for all statistical tests. 
Table 1

Ethogram used for the Current Study

\begin{tabular}{ll}
\hline Behavior & Definition \\
\hline Foraging & $\begin{array}{l}\text { Focal animal is actively engaged in acquiring or consumption of food. This included } \\
\text { food gathering, manipulating, licking, sucking, and the consumption of provided } \\
\text { food. }\end{array}$ \\
Locomotion & $\begin{array}{l}\text { Focal animal is moving actively on ground or climbing, not related to foraging. } \\
\text { autopick, defecation and urination. }\end{array}$ \\
Maintenance & $\begin{array}{l}\text { Focal animal is engaged in behavior that seems to lack purpose or involving an } \\
\text { object including somersault, body roll, hand clap, and object play. }\end{array}$ \\
Solitary Play & $\begin{array}{l}\text { Focal animal is involved in interaction with another gorilla including mother-infant } \\
\text { interactions, social groom, social play (e.g., wrestling, chasing), and aggressive acts } \\
\text { between animals (e.g., charging, displace, hit/slap, intimidation display, pull). }\end{array}$ \\
Social Behaviors & $\begin{array}{l}\text { Focal animal is resting or sleeping and not engaged in any of the previous } \\
\text { behaviors. }\end{array}$ \\
Inactive & Focal animal is engaged in regurgitation and reingestion, coprophagy, or ear- \\
covering.
\end{tabular}

\section{Results}

During the course of the six weeks, the troops' activity level ranged from $23.96 \%$ to $47.48 \%$ and troop foraging levels ranged from 8.15 to $27.87 \%$ (Figure 1). There was a significant difference in foraging and overall activity levels throughout the six weeks of the experiment (Activity: $X^{2}=14.57, N=$ 4, $p<0.05$; Foraging: $X^{2}=15.14, N=4, p<0.05$ ). Activity levels were highest with the introduction of automatic belt feeders, automatic belt feeders with additional tubes and scents, and bark boards $(46.40 \%$, $47.48 \%, 47.37 \%$ ). Similarly, foraging levels were also the highest with the presence of automatic belt feeders, automatic belt feeders with additional tubes and scents, and bark boards $(27.87 \%, 23.37 \%$, $22.24 \%)$. There were no significant differences found for abnormal behavior $\left(X^{2}=2.619, N=4, p=\right.$ n.s. $)$ which ranged between $2.18 \%$ and $4.31 \%$.

Most of the gorillas followed the same general pattern of foraging and activity levels although individual differences exist. G3 activity levels were impacted the most and reached a high of $69.19 \%$ (25.6\% increase) with the introduction of automatic belt feeders with additional tubes and scents (Figure 2). G4 was the second most impacted although his activity levels peaked at $46.9 \%$ (27.4\% increase) with the introduction of bark-boards. Although G1 showed the second lowest increase in overall activity levels, she demonstrated the largest increase in foraging with a peak at $29.0 \%$ (18.0\% increase) with the introduction of automatic belt feeders. G2 demonstrated the lowest change in foraging with a peak of $25.2 \%$ (9.9\% increase) and lowest change in overall activity levels with a peak at $43.45 \%(10.35 \%$ increase) with the introduction of automatic belt feeders. Overall foraging and activity levels for all gorillas is displayed in Figures 3 and 4. 


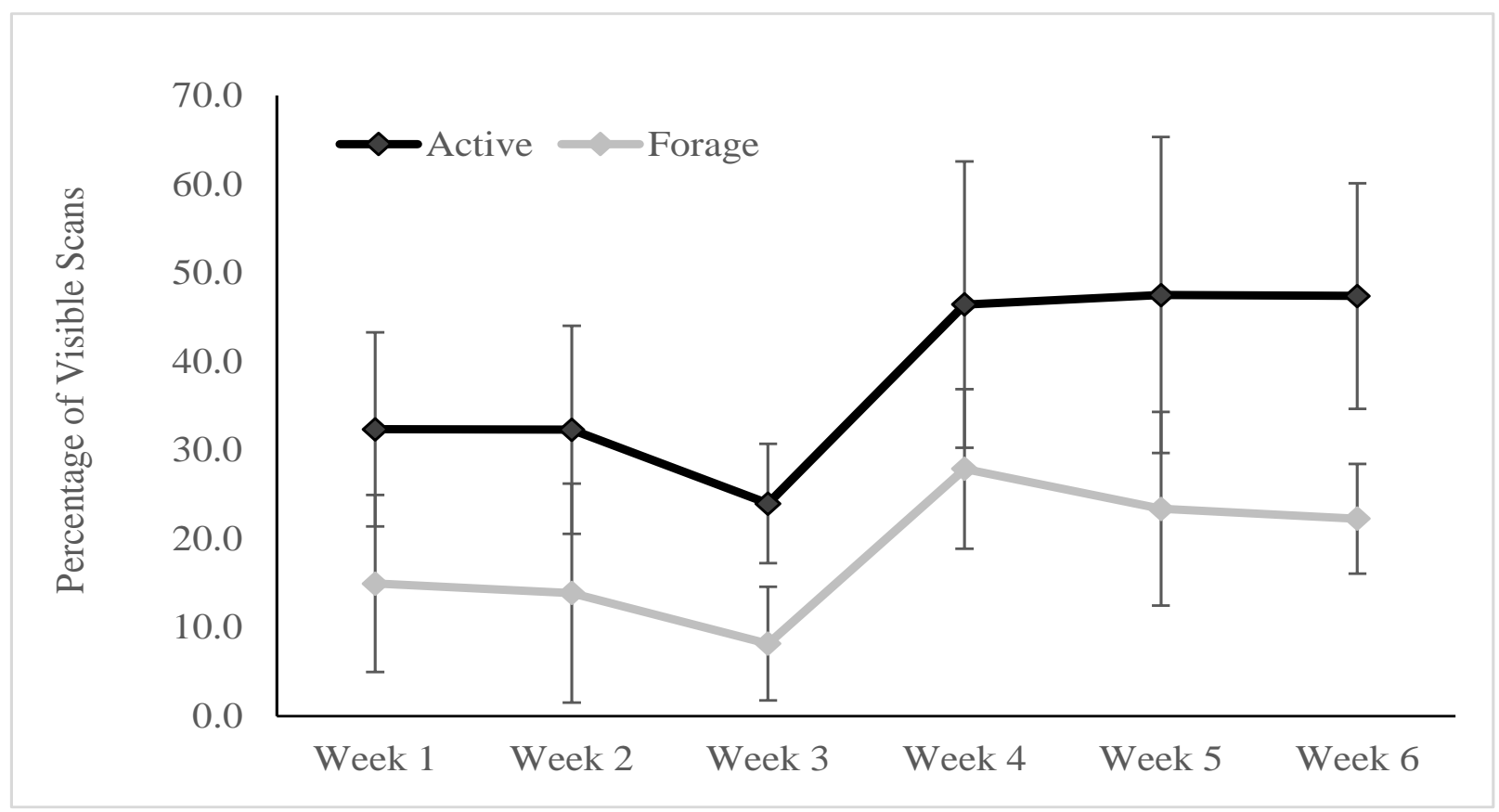

Figure 1. Weekly average foraging and activity levels throughout the study.

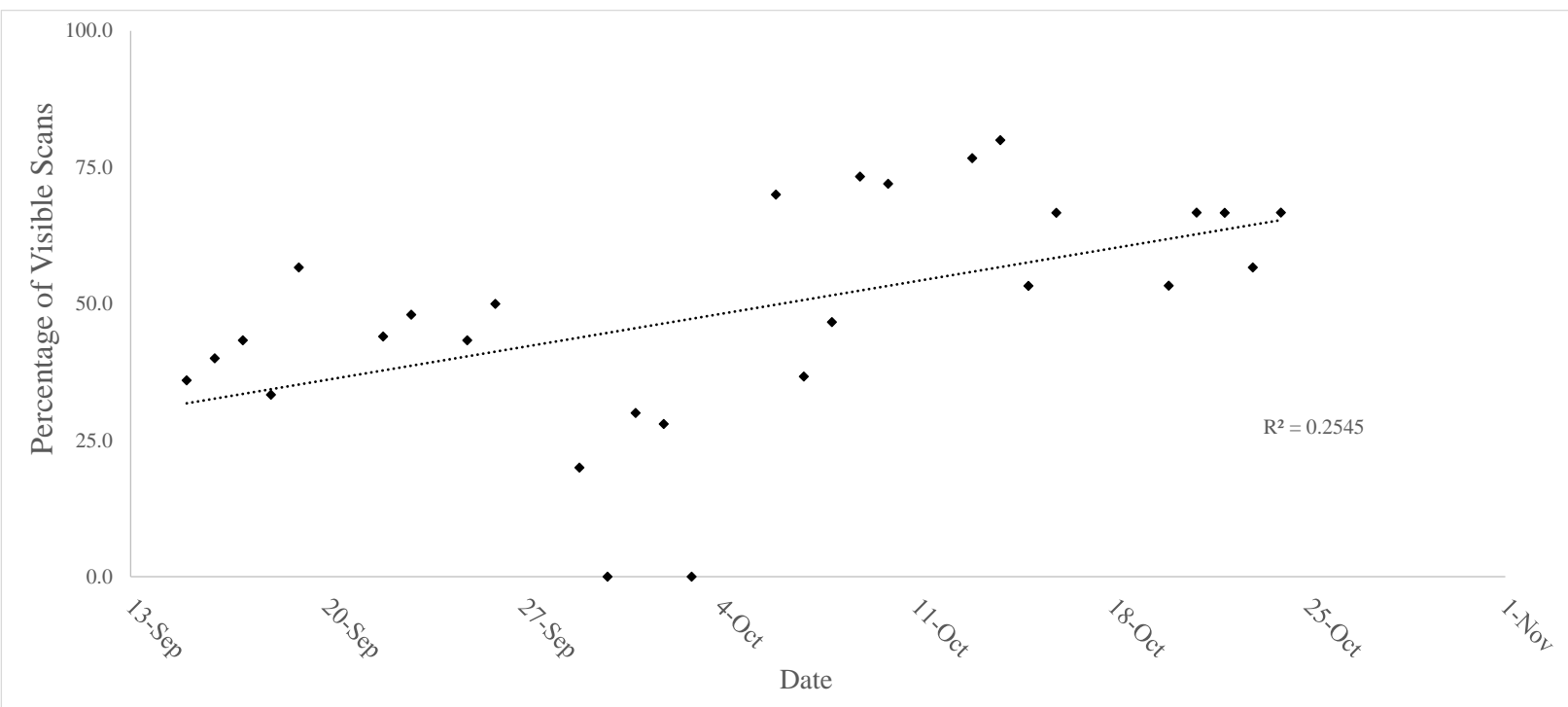

Figure 2. Daily activity levels of G1 as an example of gorillas daily activity throughout the study. 


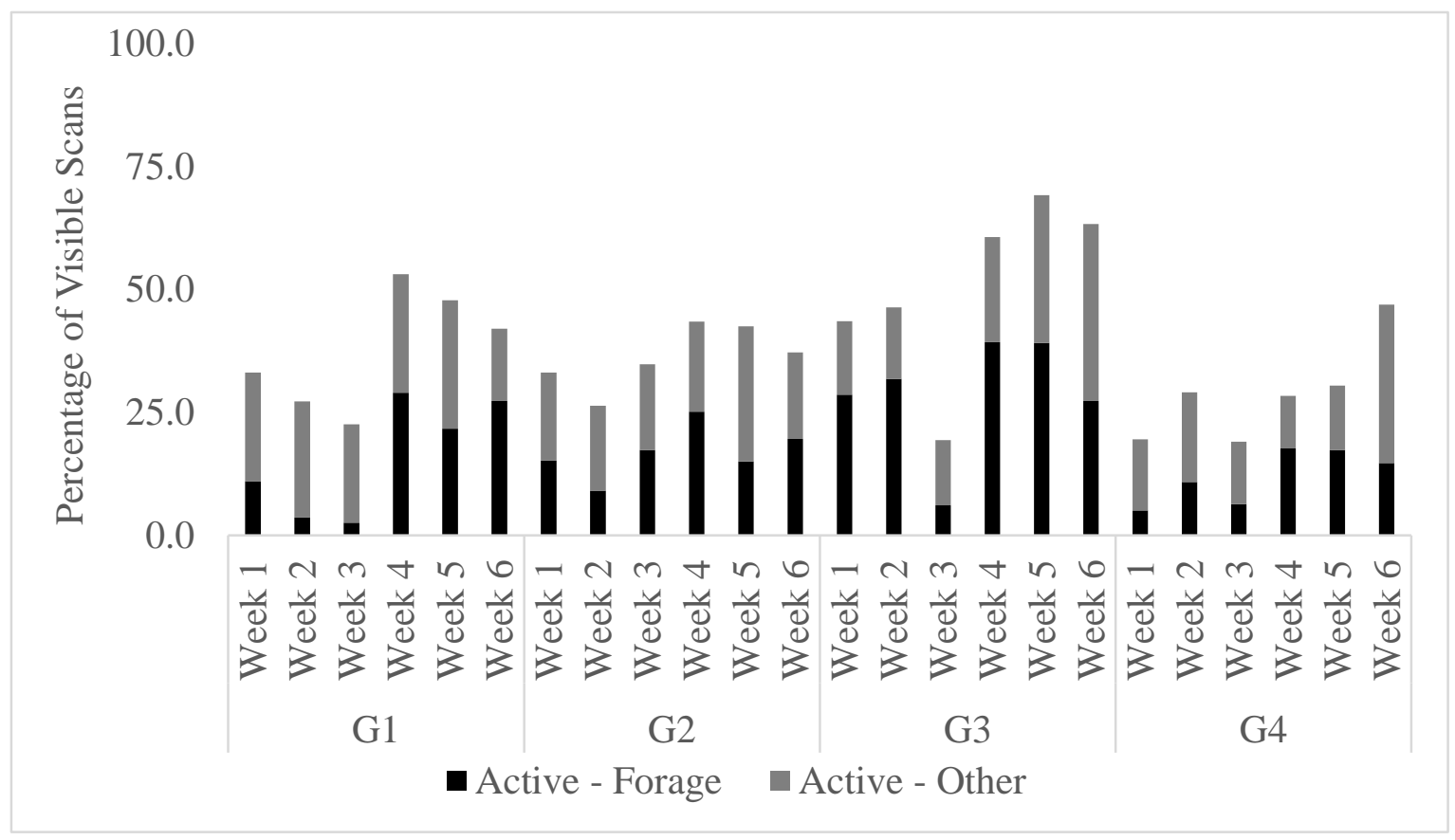

Figure 3. Individual weekly averages for foraging and activity levels throughout the study. Note. Entire bar equals the overall percentage of time spent active per week, black portion of bar includes only percentage of that time spent foraging per week.

\section{Discussion}

For this group of western lowland gorillas, various forms of environmental enrichment had a significant impact on their activity and foraging levels. With the addition of the automatic belt feeders there was the largest increase in average foraging and activity levels for the entire group. This is likely due to the unpredictability of the enrichment increasing the need to explore the environment in search of anything new. It has been suggested that the timing of enrichment can be as important as the type of enrichment (Kuczaj et al., 2002). By removing the predictability of fixed-feeding schedules and allowing for the troop to actively search for food throughout the day, it provides more opportunities for gorillas in zoological institutions to exhibit species-specific behaviors. In addition, to the randomization of time, the contents of the individual tubes deposited into the exhibit were also unknown, this added another level of unpredictability likely influencing the gorilla's behavior. However, as suggested by Watters, Miller, and Sullivan (2011), complete unpredictability of reward can be prove to have as little stimulation as complete predictability. Therefore it is possible the troop reached its threshold for activity levels with the introduction of the automatic belt feeder.

Individual differences were observed in activity and foraging levels likely due to a number of factors (e.g., type of enrichment, gender, dominance, infant, etc.). Variation in foraging times due to age and gender has been observed both within zoological institutions and the wild. Masi et al. (2009) observed that silverbacks spent approximately 20-30\% less time foraging than adult females and were observed resting 15-23\% more than adult females. These differences in foraging times were attributed to male dominance and the ability of higher ranking males to displace females from preferred feeding sites (Masi et al., 2009). Ryan, Proudfoot, and Fraser (2010) found similar results within animals in zoological institutions which is similar to the results in the current study. When food was distributed throughout the exhibit, it was observed that higher ranking animals (G2 and G4) were less able to monopolize food, thus increasing foraging opportunities for less dominant animals.

For example, the dominant silverback could guard a location of a bark board, the enrichment stump, or displace females from the training panel. This reasoning most likely explains why G1, the 
lowest ranking female, was able to benefit most from the automatic belt feeders, which were distributed throughout the exhibit. Additionally, G2 was less likely to explore the environment while attending to her infant. Therefore, enrichment which was introduced in a singular location, was utilized most by higher ranking animals. The added training session had the largest impact on G2, the dominant female, and G4, the silverback, likely due to less dominant animals wanting to avoid the enclosed area where the training panel was located.

Overall, the results suggest that providing a variety of types of environmental enrichment to gorillas within a zoological facility can increase both foraging levels and overall activity levels. Providing more opportunities to search for and acquire food allows zoo animals the ability to exhibit certain speciesspecific behaviors at higher and more natural levels. Supplying a variety of enrichment including different types and timing, can help ensure every individual animal has an opportunity to benefit. This will in turn help to make sure that every individual within the environment has an opportunity to thrive. For future research it would be beneficial to examine the use of automatic belt feeders with other species as well as the impact of increased activity levels on overall welfare.

The main goal of zoos today has transformed from a means of entertainment to education and conservation (Clayton, Fraser, \& Saunders, 2009; Reade \& Waran, 1996). Animals engaged in naturalistic behaviors has been shown to promote positive visitor experiences and conservation-learning (Clayton et al., 2009). Ensuring that animals are engaged in species-specific behavior can help ensure visitors leave with the perception that the animals are well cared for as well as an interest in supporting zoological facilities (Miller, 2012).

\section{Acknowledgements}

We thank the entire Brookfield Zoo gorilla staff for their time and efforts. We would also like to thank Alejandro Grajal, Ph.D. and F. William Zeigler for their continued support and Jay Petersen, Craig Demitros, and Cherie Barone for their assistance in making this project possible.

\section{References}

Altmann, J. (1974). Observational study of behavior: Sampling methods. Behaviour, 49, 227-266.

Clark, F. E. (2011). Great ape cognition and captive care: Can cognitive challenges enhance well-being? Applied Animal Behaviour Science, 135(1), 1-12.

Clayton, S., Fraser, J., \& Saunders, C. D. (2009). Zoo experiences: Conversations, connections, and concern for animals. Zoo Biology, 28, 377-397.

Dawkins, M. S. (2004). Using behaviour to assess animal welfare. Animal Welfare. 13, S3-S8.

Doran, D. M., \& Mcneilage, A. (1998). Gorilla ecology and behavior. Evolutionary anthropology: Issues, news, and reviews, 6, 120-131.

Hosey, G. R. (2005). How does the zoo environment affect the behaviour of captive primates? Applied Animal Behaviour Science, 90, 107-129.

Kuczaj, S., Lacinak, T., Fad, O., Trone, M., Solangi, M., \& Ramos, J. (2002). Keeping environmental enrichment enriching. International Journal of Comparative Psychology, 15, 125-137.

Less, E. H., Kuhar, C. W., Dennis, P. M., \& Lukas, K. E. (2012). Assessing inactivity in zoo gorillas using keeper ratings and behavioral data. Applied Animal Behaviour Science, 137, 74-79.

Lukas, K. E. (1999). A review of nutritional and motivational factors contributing to the performance of regurgitation and reingestion in captive lowland gorillas (Gorilla gorilla gorilla). Applied Animal Behaviour Science, 63, 237-249.

Magliocca, F., \& Gautier-Hion, A. (2002). Mineral content as a basis for food selection by western lowland gorillas in a forest clearing. American Journal of Primatology, 57, 67-77.

Masi, S., Cipolletta, C., \& Robbins, M. M. (2009). Western lowland gorillas (Gorilla gorilla gorilla) change their activity patterns in response to frugivory. American Journal of Primatology, 71, 91-100.

McPhee, M. E., \& Carlstead, K. (2010). The importance of maintaining natural behaviors in captive mammals. In D. G. Kleiman, K. V. Thompson, \& C. K. Baer (Eds.), Wild mammals in captivity: Principles and techniques for zoo management, 2nd ed. (pp. 303-313). Chicago: University of Chicago Press. 
Miller, L. J. (2012). Visitor reaction to pacing behavior: Influence on the perception of animal care and interest in supporting zoological institutions. Zoo Biology, 31, 242-248.

Reade, L. S., \& Waran, N. K. (1996). The modern zoo: How do people perceive zoo animals? Applied Animal Behaviour Science, 47, 109-118.

Rooney, M. B., \& Sleeman, J. (1998). Effects of selected behavioral enrichment devices on behavior of western lowland gorillas (Gorilla gorilla gorilla). Journal of Applied Animal Welfare Science, 1, 339-351.

Ryan, E. B., Proudfoot, K. L., \& Fraser, D. (2012). The effect of feeding enrichment methods on the behavior of captive Western lowland gorillas. Zoo Biology, 31, 235-241.

Watters, J. V., Miller, J. T., \& Sullivan, T. J. (2011). Note on optimizing environmental enrichment: A study of fennec fox and zoo guests. Zoo Biology, 30, 647-654. 